C2005 IEEE. Personal use of this material is permitted. However, permission to reprint/republish this material for advertising or promotional purposes or for creating new collective works for resale or redistribution to servers or lists, or to reuse any copyrighted component of this work in other works must be obtained from the IEEE. 


\title{
A New Functional Model for Determining Minimum and Maximum Detectable Deformation Gradient Resolved by Satellite Radar Interferometry
}

\author{
Ireneusz Baran, Mike Stewart, and Sten Claessens
}

\begin{abstract}
In this paper, a functional model for determining the minimum and maximum detectable deformation gradient in terms of coherence for synthetic aperture radar (SAR) sensors is presented. The model is developed based on a new methodology that incorporates both real and simulated data. Sets of representative surface deformation models have been simulated, and the associated phase from these models introduced into real SAR data acquired by European Remote Sensing 1 and 2 satellites. Subsequently, interferograms were derived, and surface deformation was estimated. A number of cases of surface deformation with varying magnitudes and spatial extent have been simulated. In each case, the resultant surface deformation has been compared with the "true" surface deformation as defined by the deformation model. Based on these comparisons, a set of observations that lead to a new functional model has been established. Finally, the proposed model has been validated against external datasets and proven viable. Although the major weakness of the model is its reliance on visual interpretation of interferograms, this model can serve as a decision-support tool to determine whether or not to apply satellite radar interferometry to study a given surface deformation.
\end{abstract}

Index Terms-Coherence, deformation, European Remote Sensing (ERS) satellite, interferometric synthetic aperture radar (InSAR), radar interferometry.

\section{INTRODUCTION}

$\mathbf{R}$ ADAR interferometry measures the change in range distance between the sensor and the ground. If a part of the ground covered by the imaging scene experiences a displacement $D$ along the sensor-ground direction, it changes the round trip distance by $2 D$, and creates a phase shift with respect to the stable part of the scene. Any displacement along the radar viewing direction equal to half a wavelength creates one fringe in the interferogram. Assuming that the wavelength equals $5.66 \mathrm{~cm}$ [e.g., the European Remote Sensing 1 and 2 satellite (ERS-1/2) synthetic aperture radar (SAR) sensor], one fringe corresponds to $2.83 \mathrm{~cm}$ of surface displacement.

However, not all deformations can be detected using radar interferometry. The necessary condition for deformation detection given by Massonnet and Feigl [1] implies that the maximum detectable deformation gradient is one fringe per pixel. Furthermore, Massonnet and Feigl define the maximum detectable

Manuscript received May 20, 2004; revised December 13, 2004. This work was supported by the Curtin University of Technology under an International Postgraduate Research Scholarship.

The authors are with The Western Australian Centre for Geodesy, Curtin University of Technology, Perth 6845, Australia (e-mail: I.Baran@curtin.edu.au; stewart@vesta.curtin.edu.au; claesses@ vesta.curtin.edu.au).

Digital Object Identifier 10.1109/TGRS.2004.843187

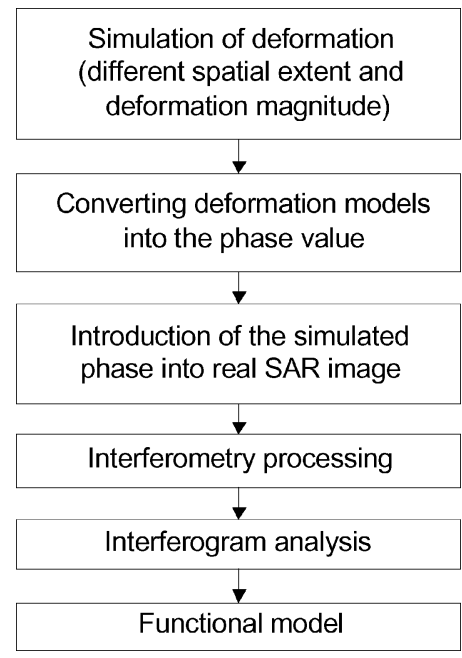

Fig. 1. Proposed methodology.

deformation gradient $\left(d_{x}\right)$ as a dimensionless ratio of half the wavelength $(\lambda)$ to the pixel size $(\eta)$

$$
d_{x}=\frac{\lambda}{2 \cdot \eta} \text {. }
$$

Thus, for the satellites ERS-1/2, the maximum detectable deformation gradient $\left(d_{x}\right)$ is equal to $1.4 \times 10^{-3}$, assuming the interferogram pixel size $20 \mathrm{~m} \times 20 \mathrm{~m}$.

According to this definition, radar interferometry should be able to detect quite a wide range of different deformation gradients. Unfortunately, radar interferometry suffers from noise in the interferogram phase measurement, which is caused by decorrelation effects [2]. Moreover, the interferogram contains biases due to satellite orbit errors, atmospheric heterogeneity [3] and filtering [4]. Hence, very small and large deformation gradients become undetectable if the level of noise is too high.

The definition of the maximum detectable deformation gradient provided by Massonnet and Feigl [1] does not include the noise factor. Thus, it does not describe the problem realistically. In addition, a definition describing the minimum deformation gradient does not exist at all. Therefore, to determine the minimum and maximum detectable deformation gradient, a new methodology is proposed. This approach consists of: 1) simulation of deformation; 2) generation of deformation related phase values; 3 ) insertion of the simulated deformation phase into a real SLC image; 4) interferometry processing; and 5) the analysis of the interferogram to estimate the signature of the recovered deformation signal, in relation to the 


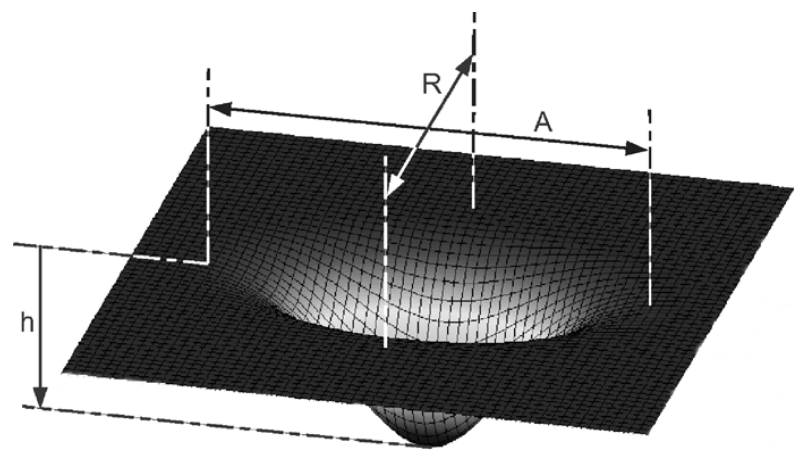

Fig. 2. Geometry of the simulated deformation model. Symbols $R$ and $A$ are the spatial extent of the model in range and azimuth, respectively.

initially simulated deformation models. Finally, an empirical set of observations that lead to the functional model defining the minimum and redefining the maximum detectable deformation gradient is determined. Fig. 1 shows the basic concept of the proposed methodology.

\section{SURfACE DEFormation Simulation}

Surface deformation may be commonly approximated as a circular or elliptical trough (e.g., mining subsidence). Thus, the two-dimensional elliptical Gaussian function has been adopted to model such a deformation

$f\left(\sigma_{R}, \sigma_{A}\right)=\frac{1}{2 \pi \sigma_{R} \sigma_{A}} \exp -\left[\frac{\left(R-\mu_{R}\right)^{2}}{2 \sigma_{R}^{2}}+\frac{\left(A-\mu_{A}\right)^{2}}{2 \sigma_{A}^{2}}\right]$.

Assuming the mean values $\mu_{R}$, and $\mu_{A}$ equal 0 , by changing the standard deviation components $\sigma_{R}$ and $\sigma_{A}$, an elliptical shape $f\left(\sigma_{R}, \sigma_{A}\right)$ for the simulated deformation is modeled. By scaling the $f\left(\sigma_{R}, \sigma_{A}\right)$, different magnitudes of vertical deformation are achieved. For simplicity, it has been assumed that vertical deformation derived from the model expresses surface subsidence along the direction of the satellite line of sight.

To simulate different deformation conditions, several models varying in spatial extent $(R, A)$ and the deformation magnitude (h), have been generated (Fig. 2). The details of the simulated deformation models are listed in Table I.

By converting (wrapping) the simulated deformation model into its associated phase values $(28 \mathrm{~mm} \Rightarrow 2 \pi)$, the change in phase $\left(\Phi_{S R}\right)$ along the radar line of sight is simulated. Such a signal is then added as a patch into the phase $\left(\Phi_{S}\right)$ of a real radar image (e.g., slave image). Thus, the phase $\left(\Phi_{S N}\right)$ of the new created image can be defined as

$$
\Phi_{S N}=\Phi_{S}+\Phi_{S R}
$$

If the absolute value of the new phase $\left(\Phi_{S N}\right)$ is greater than $\pi$, the phase is wrapped again. However, the amplitude of the real image is not changed $\left(\left|a_{N}\right|=|a|\right)$. Fig. 3 illustrates the related phase and amplitude values.

The patches are inserted to the real image over several different locations, which are characterized by different levels of noise.
TABLE I

PARAMETERS OF DEFORMATION MODELS

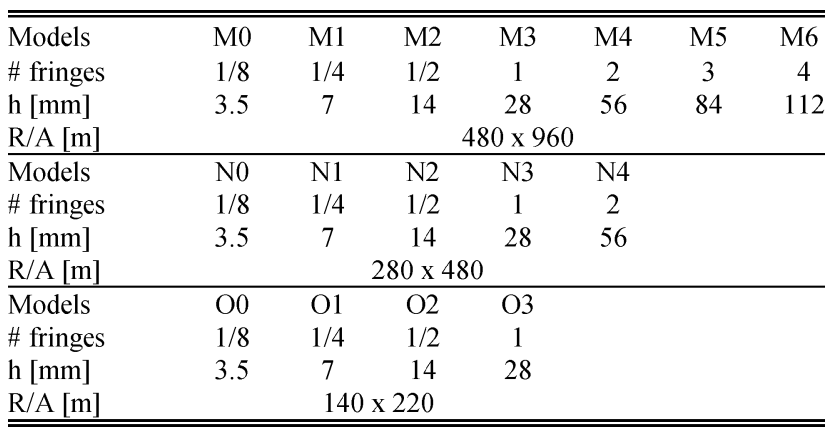

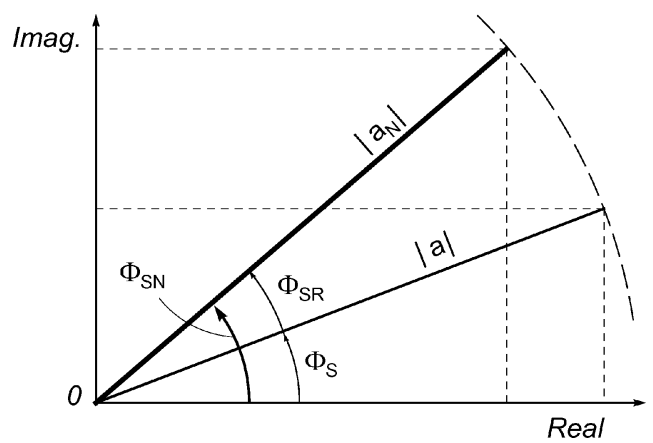

Fig. 3. Definitions of amplitude and phase for the new image in complex domain.

TABLE II

USED DATASETS OF RADAR IMAGES

\begin{tabular}{lcclll}
\hline \hline AQ-\# & Satellite & Date/Time [UT] & Track & Orbit & Frame \\
\hline AQ-I & ERS-1 & $14.11 .95 / 1: 57: 07$ & 117 & 22650 & 4164 \\
AQ-II & ERS-2 & $19.03 .97 / 1: 57: 04$ & 117 & 9991 & 4164 \\
AQ-SI & ERS-1 & $04.10 .92 / 9: 38: 05$ & 222 & 6379 & 2592 \\
AQ-SII & ERS-1 & $08.11 .92 / 9: 38: 01$ & 222 & 6880 & 2592 \\
\hline \hline
\end{tabular}

According to Hanssen and Bamler [5] decorrelations induced by the interferometry processing stages are also potential sources of noise. Therefore, the simulated phase patches are added directly into the radar image not to the interferogram. This approach ensures that interferometric processing decorrelations also contribute to the overall phase noise.

For the purpose of testing this methodology, two real radar images AQ-I and AQ-II are used (Table II). The image acquisition area is located $370 \mathrm{~km}$ north of Kalgoorlie and $700 \mathrm{~km}$ northeast of Perth in Western Australia [Fig. 4(a)]. The main advantage of choosing this area was its very stable and dry weather conditions, as well as limited vegetation cover (desert), that ensure the high quality of SAR data. Furthermore, it is located on the Yilgarn craton [Fig. 4(b)], which is recognized as a tectonically quiet area (http://www.agso.gov.au/map/). Therefore, it is assumed no signals other than the simulated deformation exist in the interferogram.

After the phase information of the simulated deformation model is introduced into the slave image, both images are processed using Delft Object-oriented Radar Interferometric 


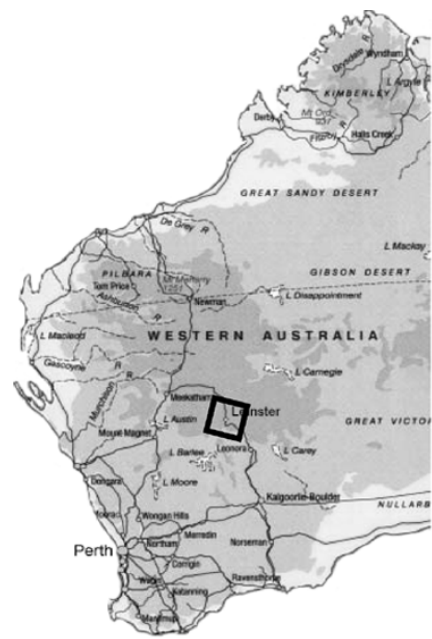

(a)

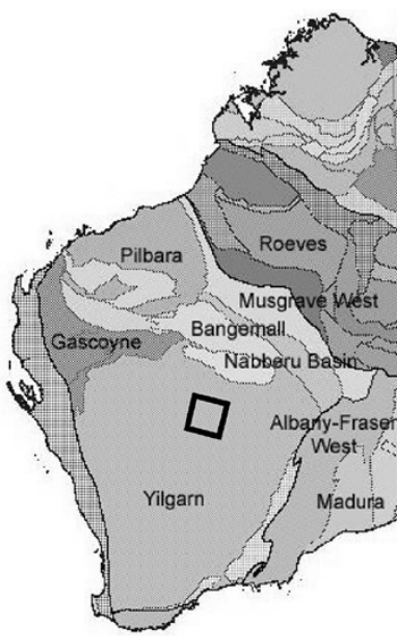

(b)
Fig. 4. (a) Location of the acquisition area and (b) map of crustal elements (cratonic regions) in Western Australia. The rectangular shape indicates the location of frame 4164 .

Software (Doris) [6] and a differential interferogram is obtained. The final interferometric phase difference $(\Delta \Phi)$ is defined as

$$
\Delta \Phi=\Phi_{M}-\left(\Phi_{S}+\Phi_{S R}\right)+n
$$

where $\Phi_{M}$ is the phase of the master image, $n$ is the noise preserved in both images as well as the noise introduced during the interferometry processing.

If the perpendicular baseline component is sufficiently short, the topography contribution can be neglected. However, in practice, it is very difficult to obtain radar images with a very small perpendicular baseline and usually the topography contribution has to be removed from the interferogram in order to obtain the signature of deformation.

The perpendicular baseline between images AQ-I and AQ-II is $195 \mathrm{~m}$. Therefore, in order to reduce the topographical effect during the interferometry processing (differential interferometry), the relevant part of the GEODATA 9 Second DEM Version 2, acquired from Geoscience Australia, has been used. The digital elevation model (DEM) is a grid of ground-level elevation points with a grid spacing of 9 arcseconds in longitude and latitude (approximately $250 \mathrm{~m}$ ). Theoretical estimates and tests of the DEM against trigonometric data distributed evenly across Australia indicate that the standard deviation of the DEM varies between 7.5 and $20 \mathrm{~m}$ for most of the continent [7]. Interferogram filtering was applied during the interferometry processing. However, it was finally omitted as it mitigated the weak deformation signal. Therefore, assuming subtracted topography contribution, (4) can be rewritten as

$$
\Delta \Phi=-\Phi_{S R}+n .
$$

According to (5), the final differential interferogram should contain the phase signal related to the simulated deformation models $\left(\Phi_{S R}\right)$ and the overall noise $(n)$. If the phase of the simulated deformation model is recovered on the differential

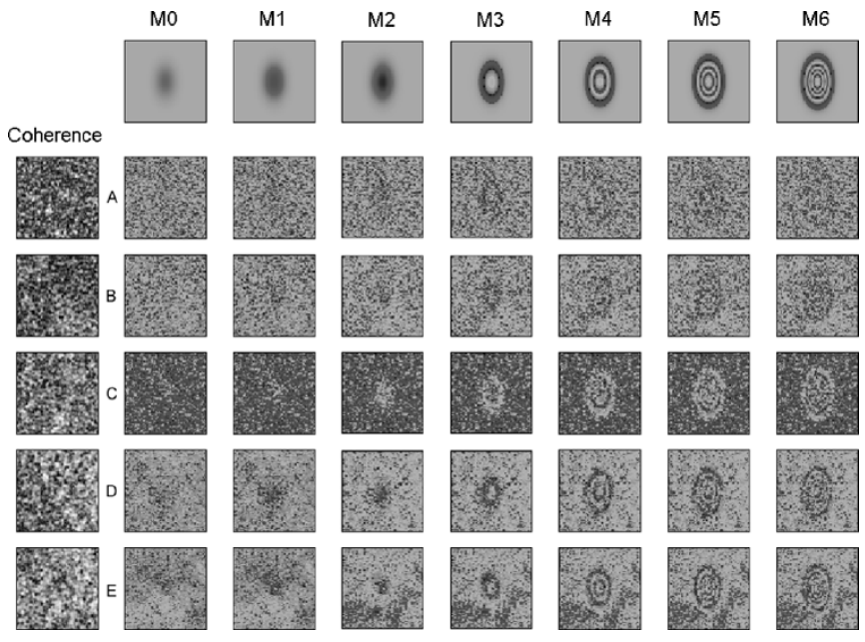

Fig. 5. Phase images of the simulated models M0 to M6 and their signatures after interferometry processing for different coherence (rows A to E). The mean values and standard deviations of the coherence subsets are (row:mean/std): A:0.30/0.10, B:0.37/0.15, C:0.41/0.14, D:0.45/0.16, E:0.51/0.15. The spatial extents of deformation are $480 \mathrm{~m} \times 960 \mathrm{~m}$. Pixel size is $20 \mathrm{~m} \times 20 \mathrm{~m}$.

interferogram, it is assumed that a particular deformation gradient can be detected.

In the following sections, the phase images of simulated deformation models are analyzed and the functional model determining the minimum and maximum detectable deformation gradient developed.

\section{Analysis of the Simulated Deformation Models}

The phase signal in the interferogram is given modulo $2 \pi$. Therefore, in order to obtain the absolute phase signal and the magnitude of deformation, the correct integer number of the whole phase cycles needs to be found. This process is called phase unwrapping. Initially, phase unwrapping was performed using Snaphu, software that uses a statistical-cost, network-flow algorithm proposed by Chen and Zebker [8]. However, due to the high level of phase noise this process was not very successful. In addition, while filtering of the wrapped phase interferogram reduced the level of noise and improved the unwrapping process it also mitigated and often destroyed the very weak deformation signal. Moreover, the majority of the deformation models represent magnitude smaller than $28 \mathrm{~mm}$, thus the interferometric phase is smaller than $2 \pi$, and there was no need for phase unwrapping. The phase images of the simulated deformation models were compared visually with the signatures from their respective wrapped phase images after interferometry processing. The visual inspection was aided by image editing software using the magnifying tool.

Figs. 5-7 show the interferometric phase of the simulated deformation models $\mathrm{M}, \mathrm{N}, \mathrm{O}$ (model parameters are defined in Table I) and the signatures of these models into the differential interferogram after interferometry processing. The phase related to the modeled deformation was introduced into the slave image over the areas characterized by different coherence values ranging from 0.3 to 0.55 .

The real interferogram based on the given radar images AQ-I and AQ-II is characterized by overall low coherence, due to the 


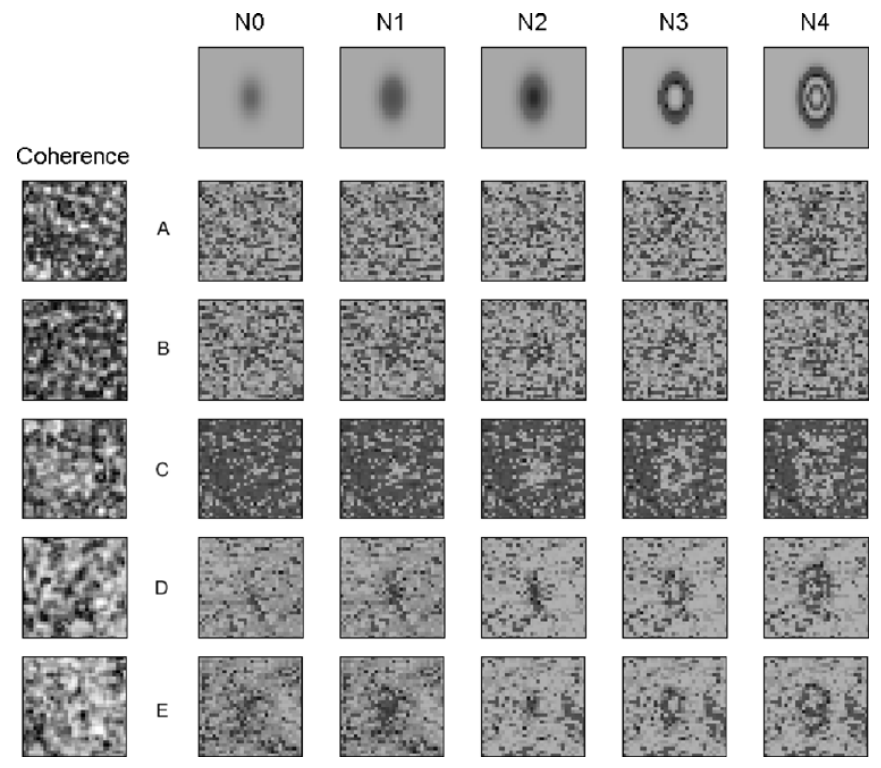

Fig. 6. Phase images of the simulated models $\mathrm{N} 0$ to $\mathrm{N} 4$ and their signatures after interferometry processing for different coherence (rows A to E). The mean values and standard deviations of the coherence subsets are (row:mean/std): A:0.30/0.13, B:0.32/0.13, C:0.42/0.14, D:0.50/0.16, E:0.54/0.15. The spatial extents of deformation are $280 \mathrm{~m} \times 480 \mathrm{~m}$. Pixel size is $20 \mathrm{~m} \times 20 \mathrm{~m}$.

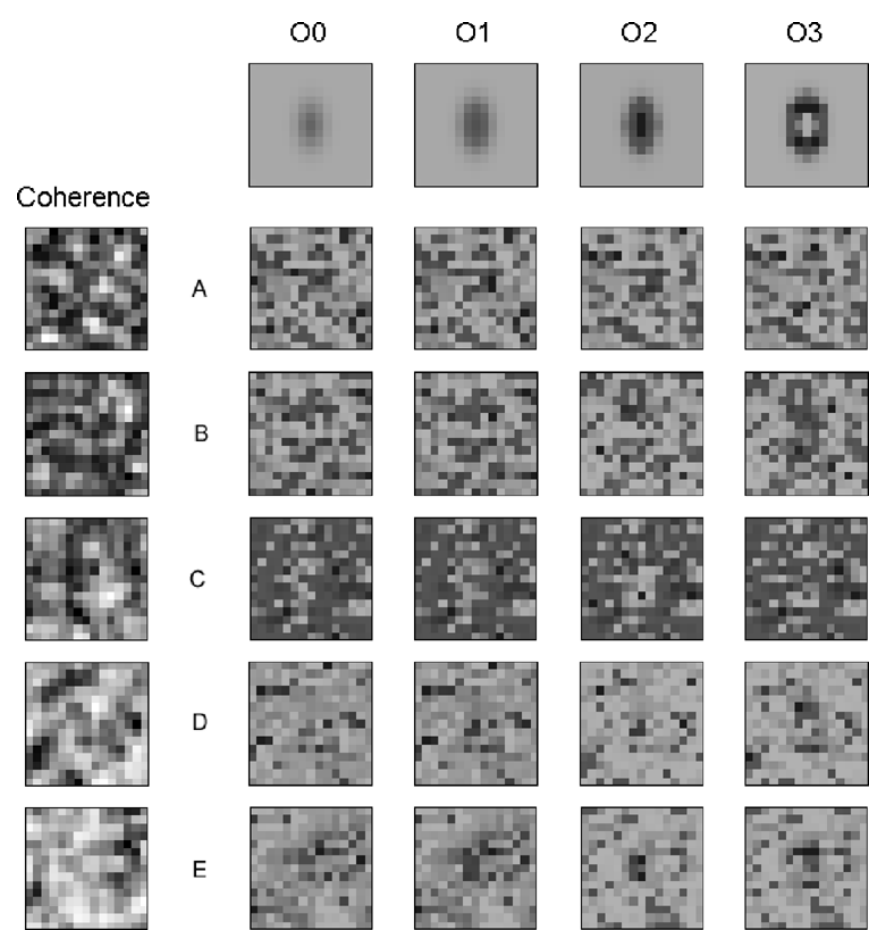

Fig. 7. Phase images of the simulated models $\mathrm{O} 0$ to $\mathrm{O} 3$ and their signatures after interferometry processing for different coherence (rows A to E). The mean values and standard deviations of the coherence subsets are (row:mean/std): A:0.30/0.13, B:0.32/0.14, C:0.40/0.14, D:0.51/0.16, E:0.56/0.15. The spatial extents of deformation are $140 \mathrm{~m} \times 220 \mathrm{~m}$. Pixel size is $20 \mathrm{~m} \times 20 \mathrm{~m}$.

long temporal baseline. This factor results in the limited range of coherence values covered. However, the covered coherence range is sufficient enough to develop a model, as the low coherence values play a crucial role in determining the model parameters.

It was found that for constant coherence, it becomes increasingly difficult to correctly interpret the interferometric phase
$(\Delta \Phi)$, as the amount of vertical deformation increases. For instance, for the interferometric phase related to models M2, M3, M4, which represent half, one, and two fringes, respectively, the number of fringes can be measured correctly for given coherence value. However, for the interferometric phase related to model "M6" (four fringes), it was not possible to unequivocally determine the number of fringes. In addition, as the increasing magnitude of deformation becomes more difficult to retrieve, the overall deformation contour pattern is more easily recognized. The extent of the deformation for the " $\mathrm{M}$ " family of models could be recognized on the interferogram for coherence values of 0.37 and above, while for models " $\mathrm{N}$," the coherence threshold was determined at the level of 0.42 (Fig. 6).

Furthermore, the smallest deformation models "O" could be detected only at coherence level greater than 0.5. Due to the small spatial extent of this model $(140 \times 220 \mathrm{~m})$, deformation could only be recognized for models $\mathrm{O} 2$ and $\mathrm{O} 3$ at coherence level 0.56 (Fig. 7).

\section{Functional Model}

In this section, a functional model defining the minimum $\left(d_{\min }\right)$ and the maximum $\left(d_{\max }\right)$ detectable deformation gradient is developed. The new model extends the definition of the maximum detectable deformation gradient by including the coherence $(\gamma)$ in its definition. Moreover, this model defines the previously undefined minimum detectable deformation gradient also in terms of coherence.

\section{A. Model Parameters}

Two parameters of the model are defined: 1) deformation gradient and 2) coherence.

The first parameter, deformation gradient $d$, is defined as

$$
d=\frac{h}{L_{\min }}
$$

where $h$ is the deformation magnitude and $L_{\min }$ is the shortest distance from the edge of the deformation model to the place where the maximum deformation magnitude occurs (Fig. 2). According to the simulated models, $L_{\min }$ is equal to half the range dimension $\left(L_{\min }=R / 2\right)$. Both variables have the same units and therefore the deformation gradient is dimensionless.

The second parameter, coherence $\gamma$, describes the interferometric phase correlation between two complex SAR images $g_{M}$ and $g_{S}$ (the interferometric pair) as defined in [9]. To estimate the coherence, the maximum-likelihood estimator given by Seymour and Cumming [10] was applied

$$
\gamma=\frac{\left|\sum_{i=1}^{N} g_{M_{i}} g_{S_{i}}^{*}\right|}{\sqrt{\sum_{i=1}^{N}\left|g_{M_{i}}\right|^{2} \sum_{i=1}^{N}\left|g_{S_{i}}\right|^{2}}}
$$

where $N$ is the number of samples within the correlation window. Coherence, in other words, is an indicator of the amount of interferometric phase noise caused by many decorrelation effects [2]. 


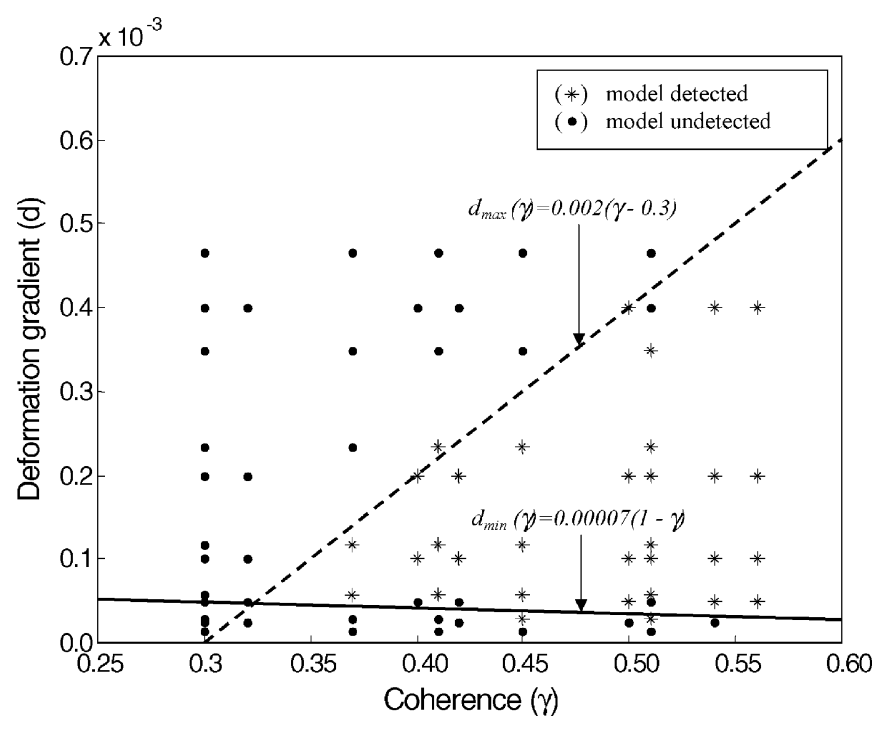

Fig. 8. Observations and the linear models: $d_{\min }(\gamma)$ and $d_{\max }(\gamma)$.

In order to develop the functional model, a set of observations based on the proposed methodology (Fig. 1) has been prepared. Each time the simulated model could be detected on the resulting interferogram, a decision value $(\Omega)$ is set to 1 . Moreover, if the model could not be detected, the decision value $(\Omega)$ is set to 0 . The correct detection of the model implies that the spatial extent as well as the correct number of fringes could be identified. The detection of the simulated deformation is undertaken by visual inspection of the interferogram. This approach is the major weakness of the resultant model. However, no automatic method that could be reliably used for accurate deformation detection analysis currently exists.

\section{B. Model Determination}

Fig. 8 shows the value of the deformation gradient $(d)$ plotted against the coherence value $(\gamma)$ based on the above observations. The dots on the plot indicate the simulated models that are not detected after the interferometry processing. The asterisks indicate the models that are properly detected after the interferometry processing. As can be seen, the point clouds related to the models that are identified as detected ( $\Omega$ equals to 1 ) form a wedge-like shape among the observations. This observation allows the definition of a model that can relate the deformation gradient and the coherence value defining $d_{\min }$ and $d_{\max }$.

To define the maximum detectable deformation gradient function $d_{\max }(\gamma)$, the observations dataset and the constraint defined in (8) have been taken into account

$$
d_{\max }=d_{x} \quad \text { for } \quad \gamma=1
$$

This constraint ensures that for a coherence value equal to one, the extended definition of the model holds for the maximum detectable deformation gradient already defined in (1). Therefore, the linear formula for the new function is defined as

$$
d_{\max }=d_{x}+S_{m}(\gamma-1)
$$

where $S_{m}$ is the slope of the linear function $d_{\max }(\gamma)$, which defines the boundary between the upper edge of the point clouds (Fig. 8).

On the basis of Fig. 8, the value of the slope $S_{m}$ was empirically determined to be approximately 0.002 . Therefore, the new definition of the maximum detectable deformation gradient in terms of the coherence value is defined as

$$
d_{\max }=d_{x}+\frac{\gamma-1}{500} .
$$

Furthermore, to define the minimum detectable deformation gradient function $d_{\min }(\gamma)$, the observations dataset and the constraint defined in (11) have been taken into account

$$
d_{\min }=0 \text { for } \gamma=1
$$

This constraint will ensure that for a coherence value equal to one, the minimum detectable deformation gradient is zero. In other words, if there is no deformation at all, the phase value should be constant. Therefore, the linear formula for the new function is defined as

$$
d_{\min }=S_{n}(\gamma-1)
$$

where $S_{n}$ is the slope of the linear function $d_{\min }(\gamma)$, which defines the boundary between the lower edge of the point clouds (Fig. 8).

On the basis of Fig. 8, the value of the slope $S_{n}$ was empirically determined to be approximately $0.7 \times 10^{-5}$. Therefore, the new definition of the minimum detectable deformation gradient in terms of the coherence value is defined as

$$
d_{\min }=-0.00007(\gamma-1)
$$

Combining (10) and (13), the functional model for determining the minimum and maximum detectible deformation gradient by radar interferometry is created.

Fig. 9 shows a graphical representation of the new functional model. The horizontal axis of the graph indicates the coherence value from 0 to 1 while the vertical axis indicates the range of possible deformation gradients from 0 to $d_{x}$. The value of $d_{x}$ is equal to $1.4 \times 10^{-3}$ and it is the old definition of the maximum detectable deformation gradient for satellite ERS-1/2 as defined in (1). Fig. 9 illustrates two zones named YES and NO. The zone YES shows the values of coherence $(\gamma)$ and deformation gradient $(d)$ for which the deformations on the surface can be detected by radar interferometry. The zone NO, however, shows the values of $\gamma$ and $d$ for which the surface deformation cannot be detected. Equation (14) defines the deformation gradient as detectable or not for certain coherence values

$$
\begin{cases}d_{\min } \leq d \leq d_{\max } & \Rightarrow Y E S \\ d>d_{\max } \text { or } d<d_{\min } & \Rightarrow N O .\end{cases}
$$

In addition, $d_{\min }(\gamma)=d_{\max }(\gamma)$, the minimum coherence value below which any deformations cannot be detected, was found to be 0.32 . 


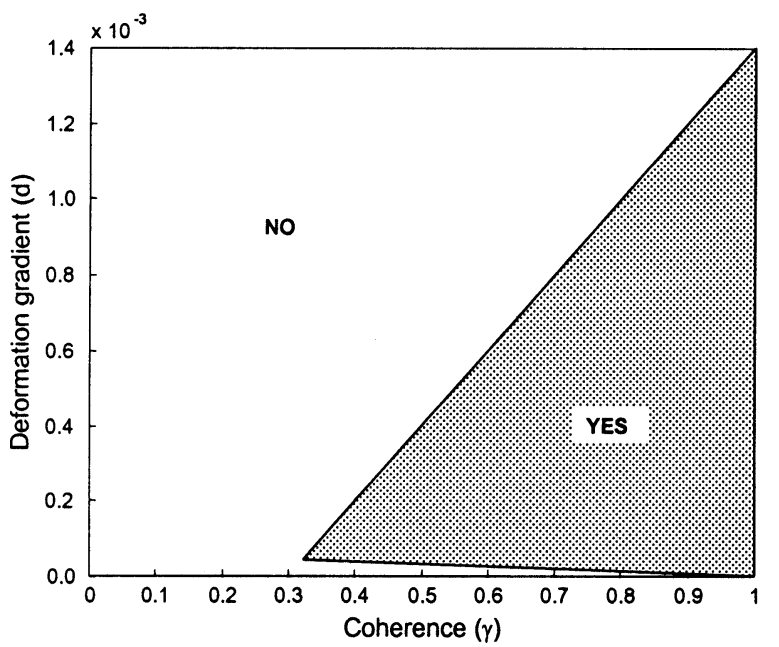

Fig. 9. New functional model for determination of the $\min / \max$ detectable deformation gradient $(d)$ in terms of coherence value $(\gamma)$. This model is valid for the satellites ERS-1/2 $(\lambda=5.66 \mathrm{~cm})$ and interferogram resolution $20 \mathrm{~m} \times 20 \mathrm{~m}$.

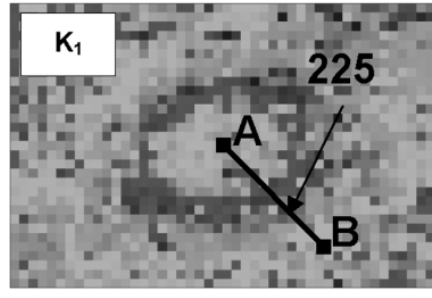

(a)

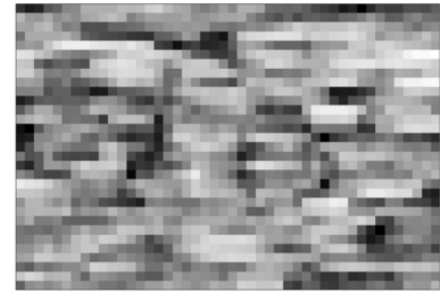

(b)
Fig. 10. (a) Deformation fringe and (b) the corresponding coherence map (mean coherence over the area is 0.61 ).

\section{VALIDATION OF THE FunCTIONAL MODEL}

The purpose of model validation is to establish whether the relationship between the model's parameters (coherence and deformation gradient) is valid for other interferometry data. To validate the proposed functional model, the interferogram from a different area based on images AQ-SI and AQ-SII is used. Since this dataset was not used for the model generation it ensures independent validation. The interferogram was processed to the final resolution $20 \mathrm{~m} \times 20 \mathrm{~m}$.

Two different areas over the interferogram to validate the functional model have been identified. Fig. 10 illustrates the first case $\left(K_{1}\right)$, which shows one fringe related to surface deformation caused by the underground mining activities. The task for this assessment is to find out if the deformation gradient and coherence taken from this example lies within the YES zone as defined by the model.

To perform this validation, two parameters of the model are established. Firstly, the deformation gradient between points A and $\mathrm{B}$ is found to be $0.12 \times 10^{-3}(0.028 / 225)$. Secondly, the average coherence over the area is found to be 0.61 . Finally, the two parameters are plotted on the graph as shown in Fig. 11. Point $K_{1}(\gamma, d)$ falls into the YES zone, which indicates that this deformation gradient can be easily detected at this coherence value. Thus, this example is in line with the proposed functional model.

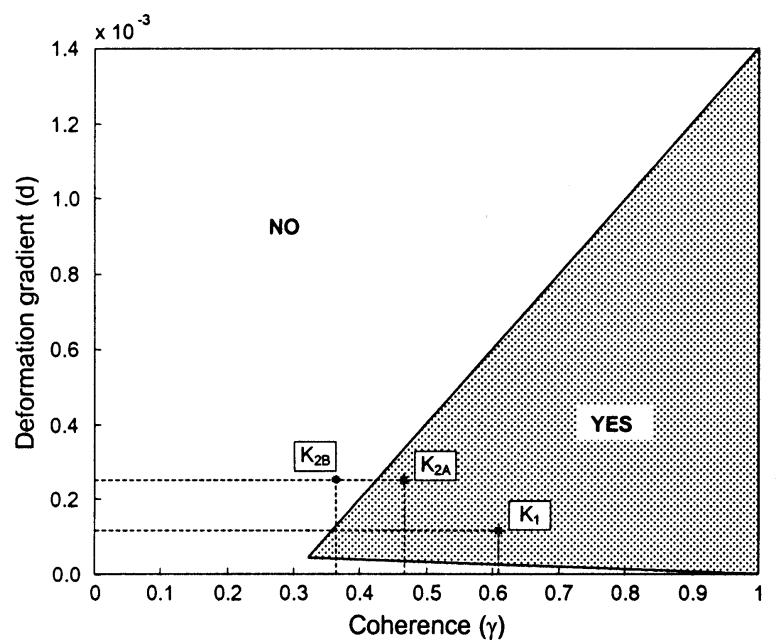

Fig. 11. Validation of the functional model. The three test cases $\left(K_{1}, K_{2 A}\right.$, $K_{2 B}$ ) agree with the proposed functional model.

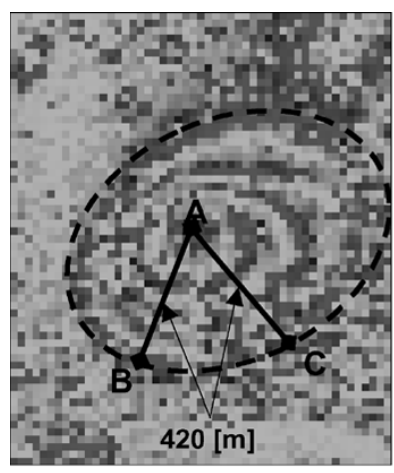

(a)

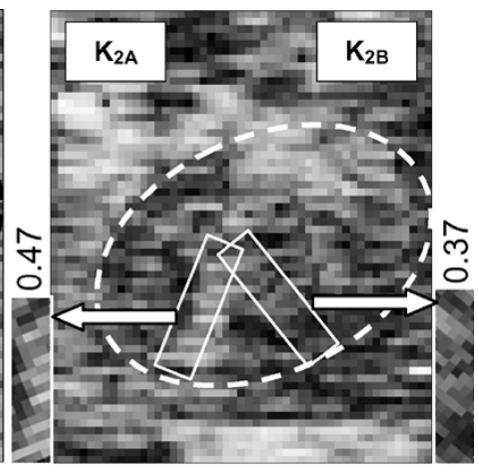

(b)
Fig. 12. (a) Deformation fringes and (b) the corresponding coherence maps.

Fig. 12 shows the second case $\left(K_{2}\right)$. This example interferogram presents four fringes in the form of an elliptical shape that are also related to surface deformation caused by the underground mining activities. However, a part of the fringes is too noisy and the correct interpretation of the number of fringes is impossible in that area. The task of this assessment is to find out if the same deformation gradient and different coherence taken from this example lies within the proper zone as defined by the model. Therefore, two subcases are selected over the area. The first subcase $K_{2 A}$ shows a part of the interferogram where the correct number of fringes can be identified and the deformation magnitude established (line AB). Subcase $K_{2 B}$ indicates a part of the interferogram where the correct number of fringes could not be identified at all (line AC). Assuming the elliptical shape of the deformation area, the deformation gradients between points $\mathrm{AB}$ and $\mathrm{AC}$ are the same in both subcases and are equal to $0.26 \times 10^{-3}$ [Fig. 12(a)].

However, the coherence value calculated over the two areas is different and equal to 0.47 in sub case $K_{2 A}$ and 0.37 in sub case $K_{2 B}$. The coherence is the average value calculated over the strips covering the investigation areas as shown in Fig. 12(b). Finally, the observed parameters related to both subcases $\left(K_{2 A}\right.$ and $K_{2 B}$ ) are plotted on the graph as shown in Fig. 11. As can be seen, the analyzed examples have further confirmed the prediction of the functional model. 


\section{CONCLUSION AND DISCUSSION}

A new methodology that leads to a functional model for determining the minimum and maximum detectable deformation gradient by radar interferometry has been proposed. This methodology is based on both simulated and real datasets. The advantage of using simulated data is that full control over the spatial extent and vertical magnitude of deformation is available. Furthermore, introducing the phase information of the simulated deformation models into a real radar image ensures realistic noise within the final differential interferogram. The resolution of the interferogram plays a very important role, especially when the purpose of interferometry is to detect deformation phenomena that has a characteristic wavelength of several hundred meters, for instance, mining related surface subsidence. Commonly used multilooking requires a compromise between reduction of the phase noise and the spatial resolution. For example, by applying the multilooking factor $5: 1$, only the resolution in the azimuth direction is reduced and a pixel of equal size $(20 \times 20 \mathrm{~m}$ for ERS- $1 / 2$ SLC data $)$ is created. This is the most common multilooking factor as it produces the best spatial resolution and uniform pixel size.

By comparing the deformation signatures on the differential interferogram with their corresponding models, a set of observations that lead to a new model has been established. The new functional model extends the existing definition of the maximum detectable deformation gradient by introducing dependence on the coherence value $\gamma$. Thus, the new definition decreases the maximum detectable deformation gradient as the coherence decreases. The new and old definitions are identical only for coherence equal to one. On the other hand, the proposed model introduces the previously undefined minimum detectable deformation gradient. It is important to notice that an increasing amount of noise also affects the ability of the interferometry technique to detect very small deformation gradients. For instance, taking into account the parameters of the satellites ERS-1/2, the maximum detectable deformation gradient is $1.4 \times 10^{-3}$ for coherence equal to one and decreases to $0.04 \times 10^{-3}$ at coherence level 0.32 . Moreover, the range of minimum detectable deformation gradients decreases from its largest value of $0.04 \times 10^{-3}$ to zero as the coherence increases to one. The zero deformation gradient can be explained as no deformation at all. Therefore, according to the conditions of the new model, radar interferometry should be able to detect deformations at their maximum detectable deformation gradient and resolve the stable areas with the maximum confidence level for the coherence value equal to one. Any coherence smaller than one would result in narrowing the range of detectable deformation gradients. Moreover, below coherence value of 0.32 , detection of any deformation is virtually impossible.

Even though the developed methodology is consistent, two weaknesses within the proposed model may be identified. These weaknesses should be taken into account when using the model in order to prevent any misinterpretations. The first weakness is the model's dependence on visual inspection. All the interferometric analyses and comparisons between phase images were based on a visual inspection of the differential interferogram. Therefore, the set of observations can be very subjective. Thus, the boundaries of the functional model are somewhat uncertain.
However, the lowest confidence level is at the lowest coherence values and increases gradually to $100 \%$ when coherence reaches one. In order to improve the confidence level at the boundaries of the model, some sort of automatic technique should replace the visual interpretation of the phase image. Such a technique could provide unbiased results. Moreover, it could extend the image interpretation and reveal additional information invisible to the human eye. RMS types of analysis or peak value of cross-correlation might provide a good measure of the line between what is detectable and what is not. Therefore, the definition of the functional model could be further adjusted. Although applying thresholds is still subjective these techniques are possible subjects for future research. The second concern is related to the spatial geometry of the modeled deformations. The simulated deformation models have a characteristic wavelength limited to several hundred meters. Although that does not cover all possible deformation modes, the resultant functional model is applicable over all characteristic wavelengths as it relies only on deformation gradient and coherence, both of which are dimensionless quantities.

Although the proposed model is very subjective, its application can still be very beneficial. By using the model, a user could find out a range of deformation gradients that can be detected assuming a given range of coherence values. Moreover, the minimum coherence value can be determined for a given deformation gradient. This can be a useful tool for the initial assessment of the suitability of the radar interferometry technique for surface deformation monitoring. The deformation gradient can be easily determined from existing knowledge about deformation geometry. The coherence could also be approximated assuming some influence of the decorrelation factors. It is well known that agricultural, forestry, urban, and desert areas as well as seasonal weather changes and time between data acquisition affect the coherence. Studies on using coherence for land-cover classification have already proved the close link between the coherence value and the land properties [11], [12]. Therefore, by reversing this approach, coherence could be estimated a priori for a particular region of interest. Having both parameters (deformation gradient and coherence) and using the model, it can be easily determined whether the surface deformation is detectable or not.

\section{ACKNOWLEDGMENT}

In-kind support was received from the European Space Agency, Geoscience Australia, and Dr. Z. Perski (University of Silesia) who supplied research data. Acknowledgment go to the three anonymous reviewers who provided valuable and constructive comments on the earlier version of the paper.

\section{REFERENCES}

[1] D. Massonnet and K. L. Feigl, "Radar interferometry and its application to changes in the earth's surface," Rev. Geophys., vol. 36, no. 4, pp. 441-500, 1998.

[2] H. A. Zebker and J. Villasenor, "Decorrelation in interferometric radar echoes," IEEE Trans. Geosci. Remote Sens., vol. 30, no. 5, pp. 950-959, Sep. 1992.

[3] H. A. Zebker, P. A. Rosen, and S. Hensley, "Atmospheric effects in interferometric synthetic aperture radar surface deformation and topographic maps," J. Geophys. Res., vol. 102, no. B4, pp. 7547-7563, 1997. 
[4] I. Baran, M. P. Stewart, B. M. Kampes, Z. Perski, and P. Lilly, "A modification to goldstein radar interferogram filter," IEEE Trans. Geosci. Remote Sens., vol. 41, no. 9, pp. 2114-2118, Sep. 2003.

[5] R. F. Hanssen and R. Bamler, "Evaluation of interpolation kernels for SAR interferometry," IEEE Trans. Geosci. Remote Sens., vol. 37, no. 1, pp. 318-321, Jan. 1999.

[6] B. Kampes and S. Usai, "Doris: The Delft object-oriented radar interferometric software," in 2nd Int. Symp. Operationalization of Remote Sensing, Enschede, The Netherlands, 1999.

[7] User Guide for the GEODATA 9 SECOND Digital Elevation Model of Australia, 2 ed., M. Hutchinson, Ed., Australian Surveying and Land Information Group and Australian National University, Canberra, 2001.

[8] C. W. Chen and H. A. Zebker, "Network approaches to two-dimensional phase unwrapping: Intractability and two new algorithms," J. Opt. Soc. Amer. A, vol. 17, pp. 401-414, 2000.

[9] R. Bamler and D. Just, "Phase statistics and decorrelation in SAR interferometry," in Proc. IGARSS, 1993, pp. 980-984.

[10] M. S. Seymour and I. G. Cumming, "Maximum likelihood estimator for SAR interferometry," in Proc. IGARSS, Pasadena, CA, 1994, pp. 2272-2275.

[11] J. M. Martinez, A. Beaudoin, U. Wegmüller, and T. Strozzi, "Classification of land-cover and forest types using multidate ERS tandem data acquired over hilly terrain," in Proc. IGARSS, Seattle, WA, 1998, pp. $1809-1811$

[12] J. Morley, J.-P. Muller, and S. Madden, "Wetland monitoring in mali using SAR interferometry,' in Proc. 'Fringe 96' Workshop on ERS SAR Interferometry, vol. 1, Zurich, Switzerland, 1997, pp. 151-166.

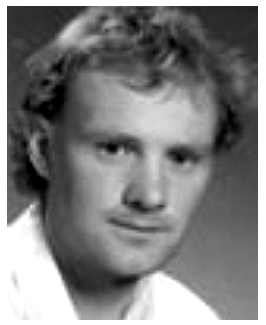

Mike Stewart was born in Manchester, U.K., in 1965. He received the B.Sc. (honoros) degree in geophysics from the University of Liverpool, Liverpool, U.K., in 1986, and the Ph.D. degree in physical geodesy from the University of Edinburgh, Edinburgh, U.K., in 1990.

$\mathrm{He}$ is currently an Associate Professor with the Department of Spatial Sciences, Curtin University of Technology, Perth, Australia. His research interests include algorithm development for GPS quality control and the application of spaceborne geodetic techniques to surface deformation monitoring.

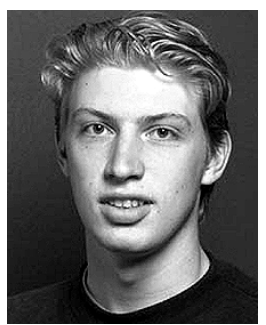

Sten Claessens was born in Maartensdijk, The Netherlands, on March 1, 1978. He received the B.Sc. and M.Sc. degrees in geodesy from Delft University of Technology, Delft, The Netherlands, in 2000 and 2002, respectively. He is currently pursuing the Ph.D. degree at Curtin University of Technology, Perth, Australia.

His primary research interests are in physical, geometrical, and satellite geodesy.

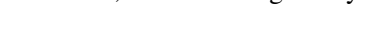

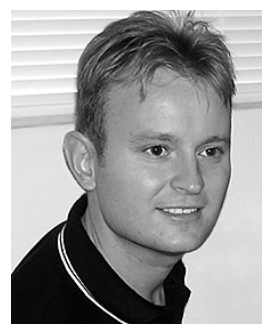

Ireneusz Baran was born in Gorlice, Poland, on September 1, 1973. He received the M.Sc. Eng. degree in surveying from the University of Science and Technology (AGH), Krakow, Poland, in 1997, and the Ph.D. degree in satellite geodesy from Curtin University of Technology, Perth, Australia, in 2004.

Since 1997, he has worked on several scientific projects including application of GPS and satellite radar interferometry to surface deformation monitoring and development of a synthetic earth gravity field model for testing regional gravimetric geoid determinations. He is currently working on GPS multipath simulation and mitigation techniques as a Research Associate in the Department of Spatial Sciences, Curtin University of Technology. His primary research interests are satellite geodesy, satellite radar interferometry, and remote sensing. 